\title{
Analysis on the LTE 800M Construction Strategy of China Telecom
}

\author{
Quqiao Wang ${ }^{1}$, Xiaolin $\mathbf{L i}^{2}$ \\ ${ }^{1}$ Application of New Technologies of Communication Research Center, Chongqing University of \\ Post and Telecommunications, Chongqing, China \\ ${ }^{2}$ Chongqing Information Technology (Group) Co., Ltd, Chongqing, China
}

Keywords: feasibility analysis, isolation buffer, simulation verification

\begin{abstract}
Combined with the early LTE hybrid network test, the Ministry of Industry and Information Technology (MII) has released LTE FDD licenses to China Telecom. LTE FDD frequency band is located in $1.8 \mathrm{G}$ band, while China Telecom's current $2 \mathrm{G} / 3 \mathrm{G}$ network are both based on $800 \mathrm{M}$ band. Firstly, this paper analyzes the feasibility of LTE FDD network construction of China Telecom CDMA 800M band, and then puts forward the co-location deployment strategy of LTE FDD and CDMA 800M. At last, the principle of setting isolation buffers between LTE FDD and original CDMA $800 \mathrm{M}$ is given, and the isolation buffer setting is simulated and verified.
\end{abstract}

\section{Introduction}

In the transition phase of "L advances and C retreats", we consider LTE re-farming construction of CDMA $800 \mathrm{M}$ band, so as to realize the efficient use of spectrum resources, thus reducing the network construction cost. In the initial stage, we consider the construction of 5M bandwidth LTE $(870-875 \mathrm{MHz})$, due to $1.4 \mathrm{M} / 3 \mathrm{M}$ bandwidth is too small to take data and voice services into account, and so there are significant deficiencies in the business balance and network interference. In the latter period, after CDMA withdraws the network, we introduce the LTE $10 \mathrm{M}$ bandwidth, thus enhancing the suburban and the rural data service performance[1]. In this context, the feasibility of LTE FDD network construction of China Telecom CDMA 800M band is analyzed, and then the strategy of LTE FDD and CDMA 800M co-location deployment is proposed. At last, the principle of setting isolation buffers between LTE FDD and original CDMA 800M is given, and the isolation buffer setting is simulated and verified[2].

\section{Feasibility Analysis of LTE Construction of China Telecom's CDMA 800M Band}

\subsection{Frequency Resources}

At present, China Telecom CDMA 800M uses the uplink and downlink 10M frequency band 7 frequency points. Frequency band uplink is $825-835 \mathrm{Mbit} / \mathrm{s}$, downlink is $870-880 \mathrm{Mbit} / \mathrm{s}, 2 \mathrm{G}$ and $3 \mathrm{G}$ use different frequency points. From the frequency band China Telecom has used, we can see that the city generally takes 6-7 frequency points, while the suburbs / rural areas occupy $2-4$. Table 1 is the usage of CDMA $800 \mathrm{M}$ band in a province. Currently 2 carrier base stations and 3 carrier base stations are still the most widely-used, accounting for $52 \%$ and $27 \%$, respectively. 
Table 1 the usage of CDMA $800 \mathrm{M}$ band in a province

\begin{tabular}{|c|c|c|c|c|c|c|}
\hline $\begin{array}{c}\text { Number of } \\
\text { Carrier } \\
\text { Wave Uses }\end{array}$ & 7 carrier & 6 carrier & 5 carrier & 4 carrier & 3 carrier & 2 carrier \\
\hline Site Scale & $2 \%$ & $4 \%$ & $6 \%$ & $9 \%$ & $27 \%$ & $52 \%$ \\
\hline
\end{tabular}

By analyzing the above data we can conclude that 52\% CDMA $800 \mathrm{M}$ base stations can free up $5 \mathrm{M}$ bandwidth for LTE FDD re-farming, and these base stations are mainly in rural areas. As $4 \mathrm{G}$ data services and follow-up VoLTE carry out step by step, 2G/3G business will gradually move back to $4 \mathrm{G}$ network, other areas can also gradually introduce LTE coverage of $800 \mathrm{M}$ based on their requirements.

\subsection{Equipment Situation}

At present, Chinese CDMA equipment manufacturers are mainly Alcatel, Huawei and ZTE. Through the research and analysis of equipment, we find that hardware design of the manufacturers are unified, so in addition to a small amount of early equipment, most devices can increase baseband board to support LTE and CDMA at the same time. The radio frequency part doesn't have much relationship with the radio frequency modulation, therefore LTE and CDMA can share the RF unit. But the current equipment is mainly 1T2R. Therefore, upgrading on the basis of the existing equipment will not be able to achieve MIMO performance, thus affecting the network downlink rate. To achieve MIMO performance, new radio frequency unit is required. The upgrading of common equipment is mainly on the basis of the same manufacturers of $2 \mathrm{G} / 3 \mathrm{G}$ and $4 \mathrm{G}$ within the region. If the manufacturers are different, a new set of equipment is needed, but the original antenna can still be shared through the combined mode[3]. Table 2 compares $4 \mathrm{G}$ network construction of different manufacturers and common manufacturers.

Table.2 4G construction contrast analysis of different manufacturers and common manufacturers

\begin{tabular}{|l|l|l|}
\hline $\begin{array}{l}\text { Comparative } \\
\text { analysis }\end{array}$ & $\begin{array}{l}\text { manufacturers of } 2 \mathrm{G} / 3 \mathrm{G} \text { and } 4 \mathrm{G} \text { are the } \\
\text { same }\end{array}$ & $\begin{array}{l}\text { manufacturers of } 2 \mathrm{G} / 3 \mathrm{G} \text { and } 4 \mathrm{G} \text { are the } \\
\text { different }\end{array}$ \\
\hline $\begin{array}{l}\text { Non-MIMO } \\
\text { mode }\end{array}$ & $\begin{array}{l}\text { Common parts: RF module, antenna, } \\
\text { feeder }\end{array}$ & $\begin{array}{l}\text { Common part: antenna, feeder } \\
\text { Upgrade part: add a set of equipment, } \\
\text { including baseband and radio frequency } \\
\text { part }\end{array}$ \\
\hline MIMO mode & $\begin{array}{l}\text { Common parts: antenna, feeder } \\
\text { Upgrade part: increase the LTE } \\
\text { baseband processing board, replace the } \\
\text { RF unit }\end{array}$ & \\
\hline
\end{tabular}

\subsection{Recommendations on Construction Strategy}

In summary, to realize a wide coverage of LTE, the use of CDMA 800M frequency resources for LTE FDD re-farming is the best choice for China Telecom. If we use $1.8 \mathrm{G}$ to realize wide coverage, a large number of sites will increase. Especially in rural and suburban areas, users are sparse, business demand is low, and investment returns are poor. Therefore, the following suggestions are proposed for China Telecom's LTE FDD network re-farming construction in the CDMA 800M band: 1. In the early stage of construction, the initial coverage of the whole network is required, and the 2 -carrier site is selected to upgrade LTE. For the areas in which manufactures of $2 \mathrm{G} / 3 \mathrm{G}$ and $4 \mathrm{G}$ networks are the same, the common equipment upgrade mode or RF module replacement upgrade mode can be used according to the business demand. For the areas in which manufactures of $2 \mathrm{G} /$ $3 \mathrm{G}$ and $4 \mathrm{G}$ networks are different, a set of LTE wireless network equipment is need, CDMA and LTE can share antenna and feeder. In the medium-term of construction, $3 \mathrm{G}$ data services gradually transfer to $4 \mathrm{G}$, while VoLTE business is carried out simultaneously, CDMA of the whole network 
leaving 2-carrier to render services to users who haven't the transfer to 4G. In the late stage of construction, migration of non- $4 \mathrm{G}$ users is completed, CDMA formally retires from network, the remaining $10 \mathrm{M}$ band can be fully covered by $800 \mathrm{M}$. For high traffic areas, a higher business experience can be achieved through CA.

\section{Co-location deployment strategy of LTE 800M and CDMA 800M}

\subsection{800M Co-channel Deployment Buffer Settings}

In the low traffic area of rural and suburban areas, the $5 \mathrm{M}$ band is separated from the $10 \mathrm{M}$ band of CDMA 800M for LTE re-farming; carrier configuration of urban base stations is high and there is no redundant band for LTE re-farming, so urban areas do not re-farm temporarily. Due to the co-channel interference problem of urban and suburban base stations, it is necessary to set isolation buffers in the suburban and rural re-farming areas, as shown in figure 1.

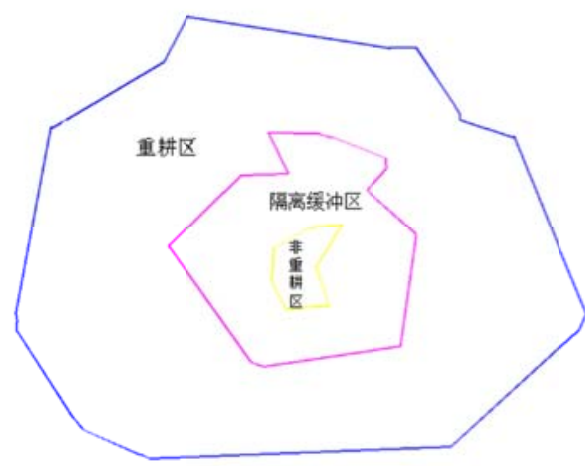

Fig. 1 schematic view of setting LTE $800 \mathrm{M}$ re-farming area and buffer

The principles of setting isolation buffers of ref-arming and not ref-arming area are as follows:

Isolation area around urban multi-carrier base stations (usually suburb) isi generally two laps of base stations, it changes with different landforms, and signaled survey results shall prevail. The isolation area is covered with a $1.8 \mathrm{G}$ base station to maintain the continuity of LTE coverage.

In particular, the width of the isolation area is estimated according to the station spacing D. Suburban station spacing D is generally $3-4 \mathrm{~km}$, the width of the isolation area should be at least greater than 2D, including 2 layers of sites. After comprehensive consideration, isolation buffers of re-farming and not re-farming area should be set to 6-8km[4].

The methods of setting isolation buffers include buffer setting method that has been simulated and verified and buffer setting method switching statistics in the background. In this paper, we adopt buffer setting method that has been simulated and verified.

\subsection{Simulation Verification of Isolation Buffer Setting}

We use the method of system simulation to verify whether the isolation buffer setting is reasonable. Simulation conditions: in re-farming and not re-farming area base stations, the antenna height of CDMA and LTE is set to $25 \mathrm{~m}$, and the transmission power is set to $20 \mathrm{~W}$. As it is shown in figure 2, simulation coverage results are the coverage radius corresponding to the following received power. LTE downlink reach RSRP of CDMA terminal: $-113 \mathrm{dBm},-103 \mathrm{dBm}$; CDMA downlink signal reach Rx of LTE terminal: $-107 \mathrm{dBm},-95 \mathrm{dBm}$. 

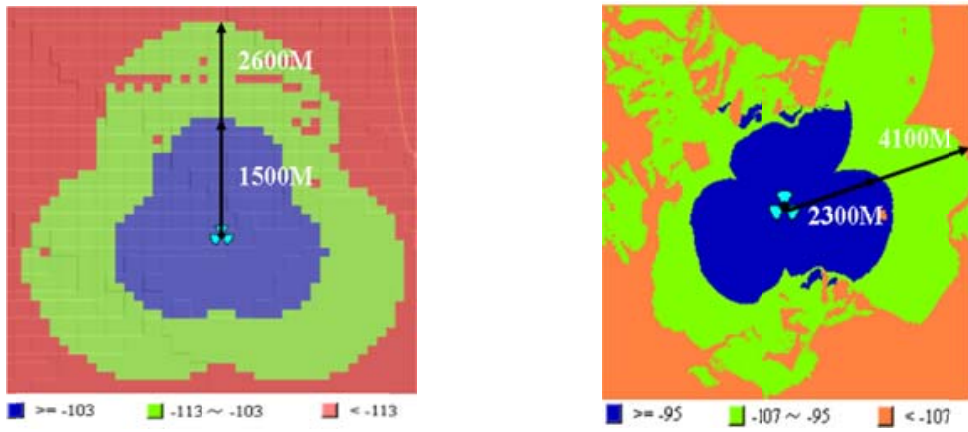

Fig. 2 coverage radius of LTE $800 \mathrm{M}$ and CDMA $800 \mathrm{M}$ base station

Taking an area as an example, we use Shanghai Bailin simulation software to simulate not re-farming area and re-farming area. Figure 3 shows the RSRP simulation of LTE $800 \mathrm{M}$ in re-farming area and Rx simulation of CDMA $800 \mathrm{M}$ in not re-farming area. We can conclude that LTE $800 \mathrm{M}$ signal of re-farming area won't interference base stations in not re-farming area. Neither will CDMA 800M signal of not re-farming area interference base stations in re-farming area.

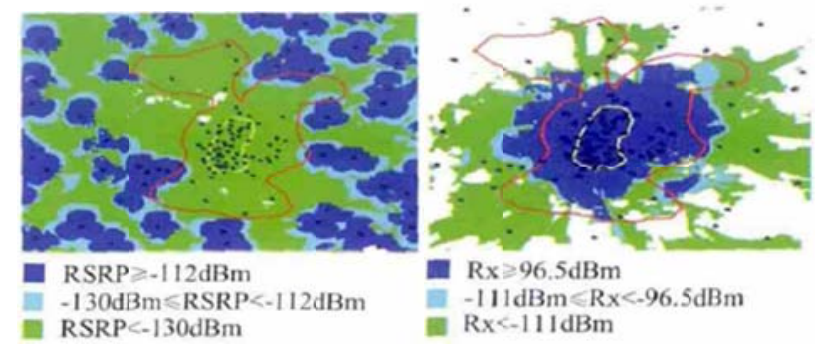

Fig. 3 isolation buffer zoning simulation results

As can be seen from the verification results, the isolation buffers setting between not re-farming area and re-farming area is appropriate.

\section{Conclusion}

Based on the above analysis, it is feasible for China Telecom to take advantage of CDMA 800M for LTE FDD network construction. Based on the network construction process and VoLTE development, operators can gradually promote the relevant constructions. As to current situation, the main difficulties lie in the industrial chain. It is necessary for operators and manufacturers to promote the large-scale production of the main equipment, as well as the research and development of $2.1 \mathrm{G}, 1.8 \mathrm{G}$ and $800 \mathrm{M}$ multi-mode multi-frequency terminals.

\section{References}

[1] M. Porjazoski and B. Popovski, "Contribution to analysis of Intercell interference coordination in LTE: A fractional frequency reuse case," Mobile Congress (GMC), 2010 Global, Shanghai, 2010, pp. 1-4.

[2] Y. Kolyadenko and A. Alali, "Intersystem interference on LTE network bandwith influence analysis," Problems of Infocommunications Science and Technology, 2014 First International Scientific-Practical Conference, Kharkov, 2014, pp. 125-126.

[3] W. Li, J. Chen, H. Long and B. Wu, "Performance and Analysis on LTE System under Adjacent Channel Interference of Broadcasting System," Computer and Information Technology (CIT), 2012 IEEE 12th International Conference on, Chengdu, 2012, pp. 290-294

[4] JIN Z J. Discussion on LTE wireless network optimization[J]. Mobile Communications, 2014(38): 9-13. 